\title{
Degeneración tumoral en meningocele no intervenido. Descripción de dos casos
}

\author{
D. Rivero-Celada; L.I. Carcavilla-Loncán; M.A. Marín-Cárdenas*; J.M. Cantero-Antón; J. Alfaro-Torres**; F. Duato- \\ Jané*** y J. Eiras-Ajuria
}

Servicio de Neurocirugía. Unidad de Neurorradiología*. Servicio de Anatomía Patológica**. Servicio de Cirugía Plástica***. Hospital Universitario Miguel Servet. Zaragoza.

Resumen

Asistir a pacientes que presentan un meningocele gigante y en edad adulta es una rareza clínica, debido en parte a que es una malformación disráfica con una incidencia baja y a que el índice de supervivencia de los recién nacidos con éstas patologías era menor en épocas anteriores, pese a la benignidad de la malformación.

Aportamos a la literatura existente los casos de dos pacientes que nacieron con un disrafismo espinal abierto y que no fueron operados en su momento, llegando a la edad adulta con voluminosos sacos meningocutáneos que tuvieron que ser extirpados, por presentar en el caso de más edad, un absceso con fistulización sin salida de LCR que no mejoró con tratamiento tópico y antibiótico. En el otro caso por sospecha de malignización debido a crecimiento progresivo de la malformación durante el último año, endurecimiento de la piel y dolor.

El estudio anatomopatológico de los sacos resecados demostró la existencia de una degeneración carcinomatosa.

En nuestros casos y en los pocos casos encontrados en la bibliografía parece que la irritación crónica del LCR y la existencia de células multipotenciales en el saco meningocélico pueden favorecer la malignización de los tejidos que recubren el mismo.

La posible malignización descrita en la escasa literatura existente, a la que aportamos nuestros dos casos, sugiere como tratamiento de elección la exéresis quirúrgica de estas lesiones congénitas lo más precozmente posible

PALABRAS CLAVE: Meningocele. Espina bífida. Degeneración carcinomatosa. Sarcoma.

Tumoral degeneration occurring over a non-healing meningocele. Report of two cases

Recibido: 6-06-05. Aceptado: 26-01-06

\section{Summary}

A giant meningocelic sac has not been usually described in adult patients, due to the fact that it shows a low incidence and few newborn have survived to date though the malformation is benign.

We report two cases of patients born with the described malformation and who were not operated at that time, so they reached adulthood with bigger sacs. They needed surgery to remove the sacs, for a different reason. The older one had a fistulous abcess but the LCR did not come out, and it did not improved by the application of topic and antibiotic treatment. The other patient showed a progressive growth of the malformation during the last year, skin hardening and pain.

The histological study of the dried sacs proved the existence of a carcinomatous degeneration.

In the patients we have treated, it seems that a chronic irritation of the LCR and the appearance of multipotent cells in the meningocele may favour the malignancy of the tissues surrounding the sac.

This possible malignancy, already described in the bibliography, suggests a prompt elective surgical treatment of the patients with these congenital lesions as soon as possible.

KEY WORDS: Meningocele. Spina bifida. Carcinomatous degeneration. Sarcoma.

\section{Introducción}

La placa neural es la estructura embrionaria responsable de la formación y desarrollo del sistema nervioso en el embrión. A lo largo de su desarrollo, esta estructura pasa por diversas transformaciones hasta convertirse en el tubo neural y cualquier alteración durante su cierre conllevará la aparición de la espina bífida ${ }^{6,18}$.

La evolución y desarrollo del eje neural se produce durante tres fases de la embriogénesis: Fase I ó de neurulación, fase II ó de canalización y fase III ó de diferenciación regresiva; ésta última dura hasta el nacimiento. 
El meningocele constituye un tipo de disrafismo espinal quístico que consiste en un defecto en el cierre de los arcos vertebrales y distensión de las meninges en forma de quiste conteniendo únicamente LCR, sin tejido neural en su interior. Este disrafismo se produce durante la fase de neurulación, hacia el final de la cuarta semana, y dentro de ésta, coincidiendo con el cierre del neuroporo posterior, en el día 26 del desarrollo embrionario ${ }^{3}$.

Clínicamente se presenta como una tumoración posterior blanda sobre el raquis, que exterioriza y fija la parte posterior del saco dural que se encuentra recubierto total o parcialmente por la piel; puede estar abierto o cerrado.

En algunos casos el meningocele puede acompañarse de otras malformaciones del SNC, apareciendo entre otras la medula anclada, "tigh fillum", diastematomielia, el quiste epidermoide, el lipoma y la hidromielia como las más comunes. No obstante, las dos malformaciones más frecuentes e importantes que se asocian a la espina bífida son la hidrocefalia y la malformación de Chiari II ${ }^{5,13}$.

La incidencia mundial de espina bífida es de 1-2/1000 nacidos vivos (0'1-0'2\%). La prevalencia en la población española es de 1'01 por 1000 nacimientos, frecuencia que puede considerarse como de media-baja ${ }^{19}$.

Se ha podido demostrar una incidencia familiar aumentando el riesgo entre un 2-3\% si existe antecedente de un hijo previo con espina bífida, llegando a ser del 6-8\% después de dos hijos afectados. También aumenta en familias con parientes cercanos que han tenido niños con disrafismo espinal, especialmente si son por línea materna, en épocas de guerra o en periodos de hambre ${ }^{9,17}$. La transmisión genética corresponde a patrones no mendelianos, probablemente de tipo multifactoriales.

Se conocen múltiples factores etiológicos y se ha relacionado con estados deficitarios de ác. fólico y zinc, con la hipervitaminosis A y con fármacos teratógénicos, como la carbamacepina y el ác. valproico. Los síndromes febriles y virásicos durante el embarazo también se han implicado como factores de riesgo ${ }^{16}$.

El objeto de nuestra comunicación es mostrar la tendencia a la malignización de los tejidos que recubren el saco meningocélico en los dos únicos pacientes que hemos encontrado en nuestro centro, portadores del meningocele en edad adulta. Se ha realizado una búsqueda bibliográfica de casos similares al nuestro y se ha constatado la ausencia de literatura al respecto.

\section{Material y métodos}

Presentamos los casos de dos pacientes adultos, recogidos a lo largo de los 36 años de historia en nuestro Servicio Regional de Neurocirugía, que acudieron a nuestras consultas por presentar en los últimos meses, cambios en los sacos meningoteliales lumbares de gran tamaño que tenían

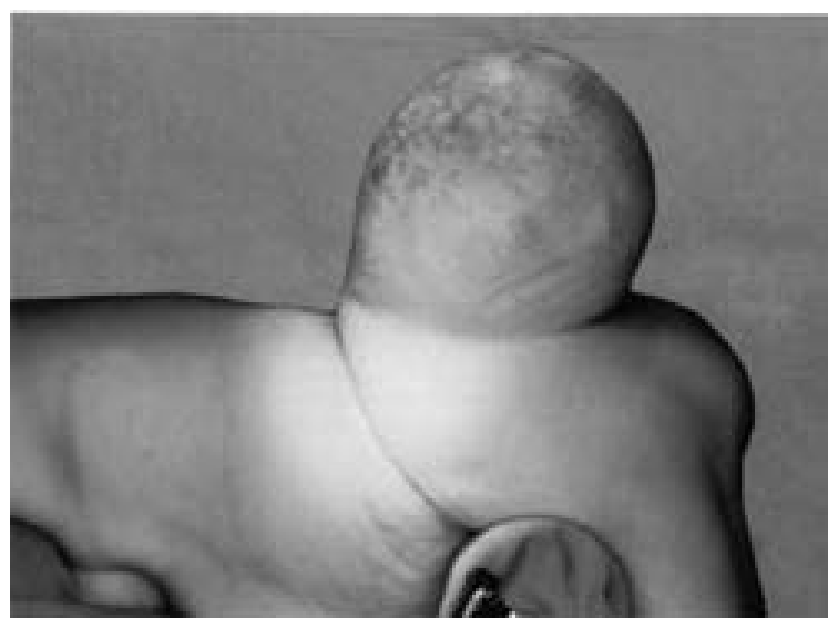

Figura 1. Imagen del bultoma lumbar.

desde su nacimiento. Ninguno antes había sido tratado mediante cirugía.

\section{Caso 1}

Paciente atendida en nuestras consultas en 1979.

Mujer de 53 años, madre de 3 hijos que había realizado una vida normal.

Desde que nació, había convivido con un bulto en región lumbar que, a pesar del aspecto estético, no limitaba su actividad diaria habitual y nunca había tenido manifestaciones clínicas. La ausencia de sintomatología y el riesgo quirúrgico, fueron las causas por las que inicialmente los médicos no indicaron a sus padres un tratamiento quirúrgico.

Pasados bastantes años, tampoco pensó en resecar su lesión, tal vez influenciada por el miedo o, simplemente, porque no lo consideraba necesario.

No relataba enfermedades previas de interés ni recordaba haber drenado líquidos por su malformación.

Durante el último año notó un crecimiento progresivo de su lesión, que le obligó a aumentar las tallas en sus vestidos. Se acompañaba de una sensación de tensión y de endurecimiento cutáneo, con dolor al comprimirlo cuando se sentaba o en la cama. Como el dolor progresivamente iba en aumento y precisaba cada vez de más analgesia, aceptó que su médico la remitiera a nuestras consultas.

Presentaba una voluminosa masa lumbar completamente epitelizada de $20 \times 15 \times 12 \mathrm{~cm}$., discretamente tensa con zonas al tacto más irregulares y endurecidas, dolorosa a la presión, sin evidencia de signos inflamatorios (Fig. 1). La exploración neurológica fue normal y los estudios analíticos generales se encontraron dentro de la normalidad.

La radiología simple de raquis lumbar mostró defecto de fusión del arco y láminas de L5 y S1. Un estudio 

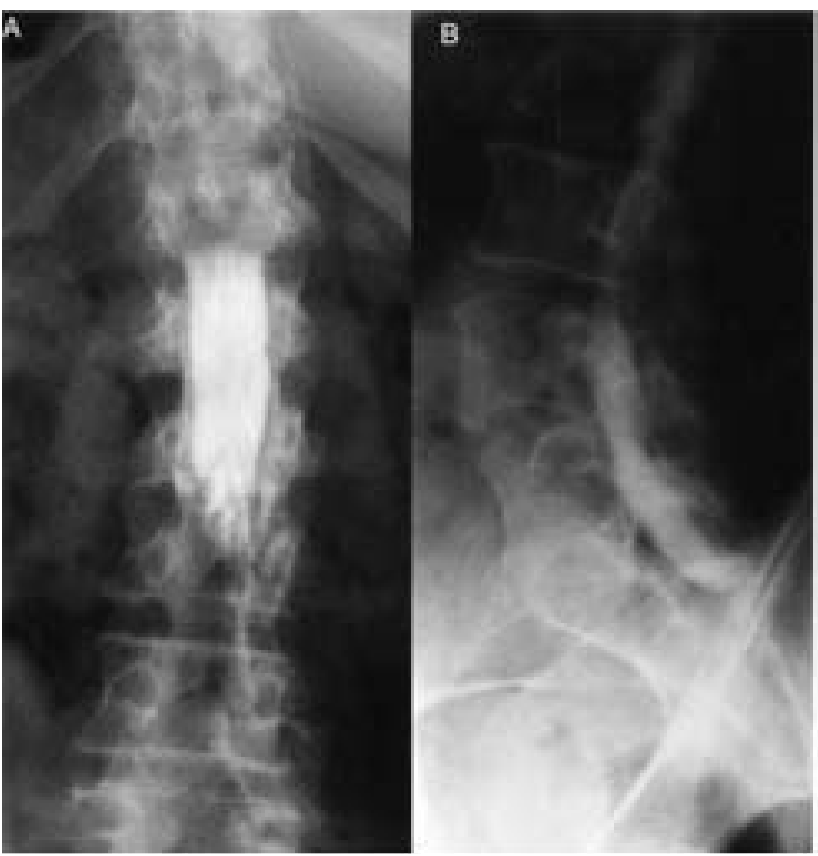

Figura 2. (A) Mielografía en proyección AP: contraste radiopaco detenido en L3- L4 por la deformidad del saco dural en la proximidad de la malformación; defecto de fusión de L5. (B) Proyección lateral oblicuada con deformidad de la columna de contraste sin comunicación con el saco.

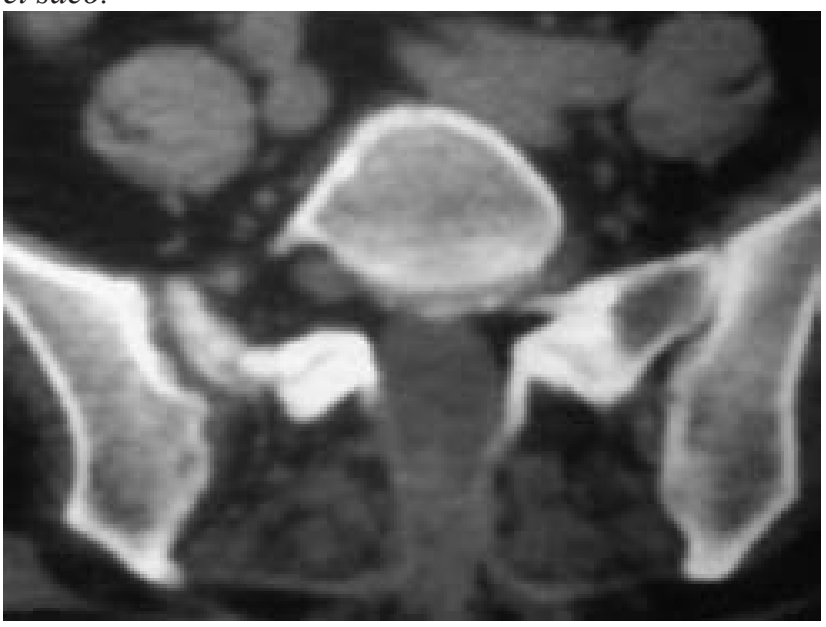

Figura 3. T.A.C. lumbar: Corte axial sobre S1. Defecto de cierre del arco posterior. Masa en partes blandas que comunican con el canal raquídeo a través de la línea media posterior.

mielográfico puso de manifiesto retención del contraste a nivel L3 y L4 por deformidad del saco dural previo al disrafismo, pero sin continuidad con el saco del bulto (Fig. 2). El estudio de TAC confirmaba este defecto y ponía de manifiesto la existencia de un tracto de continuidad desde el canal raquídeo con una masa exteriorizada y localizada en partes blandas (Fig.3).

Los cambios clínicos comentados obligaron a proponer a la paciente la necesidad de un tratamiento quirúrgico, que aceptó.

Bajo anestesia general, se resecó quirúrgicamente el bulto en bloque, confirmándose la existencia de un tracto duro y fibroso basal que continuaba y se introducía por línea media a través del defecto espinal. Se seccionó este conducto en su entrada a nivel de la fascia muscular y, tras comprobar que no existía fístula, no se consideró necesario continuar la intervención. El defecto cutáneo se reparó directamente con la propia piel del saco.

El estudio anatomopatológico indicó que se trataba de un tejido con componente celular mixto de meningosarcoma y de teratocarcinoma. La paciente falleció unos meses después por una neumonía, sin poder precisar su etiología.

\section{Caso 2}

Paciente remitido a nuestras consultas de neurocirugía en 2004.

Varón de 72 años, padre de 2 hijos. Siempre realizó una vida sin limitaciones incluida la realización del servicio militar. Nunca, ni de niño, recordaba haber tenido molestias relacionadas con su bultoma lumbar. Conocía cómo se llamaba y en qué consistía su lesión desde muy joven; años más tarde, al ser valorado en una revisión de rutina laboral, le indicaron de nuevo que su malformación consistía en un meningocele L3-L4 con raquisquisis desde L3 a L5. No recordaba haber tenido salida de secreciones por el saco.

Por causas semejantes al caso anterior nunca se quiso operar.

Como enfermedades previas de interés relataba haber tenido, los 63, años un cuadro de ACV que dejó como secuela una ligera hemiparesia izquierda. A los 70 años ingresó de urgencia en el servicio de Neurología de este hospital por presentar un cuadro de instauración súbita, de paraplejia completa, mientras paseaba, con nivel sensitivo D8-D9. Se realizaron los estudios pertinentes que confirmaron que se trataba de un proceso isquémico medular, del cual no se recupero funcionalmente.

Se remitió a nuestro servicio en la fecha ya indicada, por presentar un cuadro de infección abierta con salida de secreción purulenta del saco meningocélico de 3 semanas de evolución que no había cedido a la antibioterapia y cuidados instaurados en su domicilio. No presentaba ningún signo de meningismo. En la exploración se apreciaba un saco cutáneo en región lumbar con un tamaño de $10 \mathrm{x}$ $6 \times 10 \mathrm{~cm}$., abierto con salida de pus, sin salida licuoral (Fig.4).

Se intentó de nuevo en nuestro medio un tratamiento 


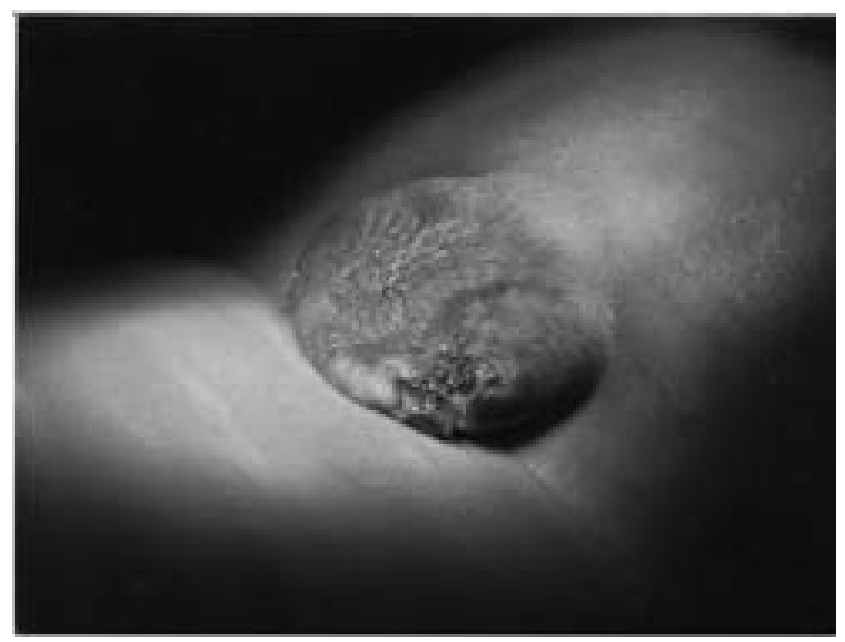

Figura 4. Imagen del meningocele abierto.

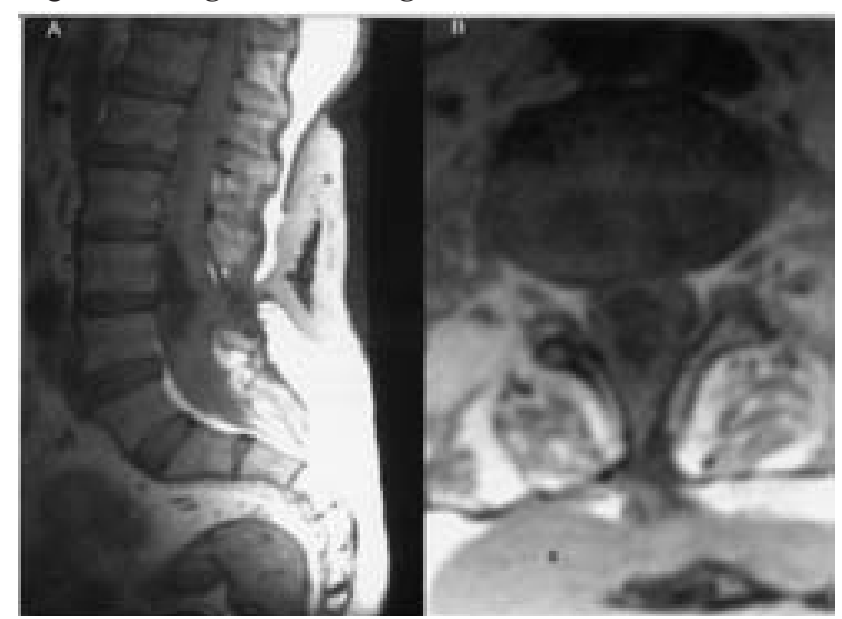

Figura 5. RMN (A) parasagital y (B) axial potenciadas en $T 1$ de raquis lumbar. Defecto de cierre del arco posterior y comunicación del saco dural con una masa exteriorizada en partes blandas sin contenido neural(s). En el interior del canal se observa un contenido sólido que pudiera tratarse de un tejido de descamación o bien de tejido embrionario junto con señal de LCR.

conservador con antibioterapia específica, limpieza y lavados del saco sin lograr ninguna mejoría, por lo que se planteó la resección quirúrgica del mismo. La radiología simple de raquis lumbar mostraba raquisquisis a nivel L3-L4. El estudio de RNM confirmaba este disrafismo y mostraba un tracto fibroso que comunicaba el saco dural con una masa localizada en partes blandas, con exteriorización cutánea (Fig. 5).

Bajo control anestésico general, se resecó quirúrgicamente el saco malformativo confirmándose la persistencia de un tracto fibroso en su base que penetraba y continuaba a través del disrafismo de la línea media hacia el interior. Se seccionó a su entrada sobre la fascia muscular lumbar y tras

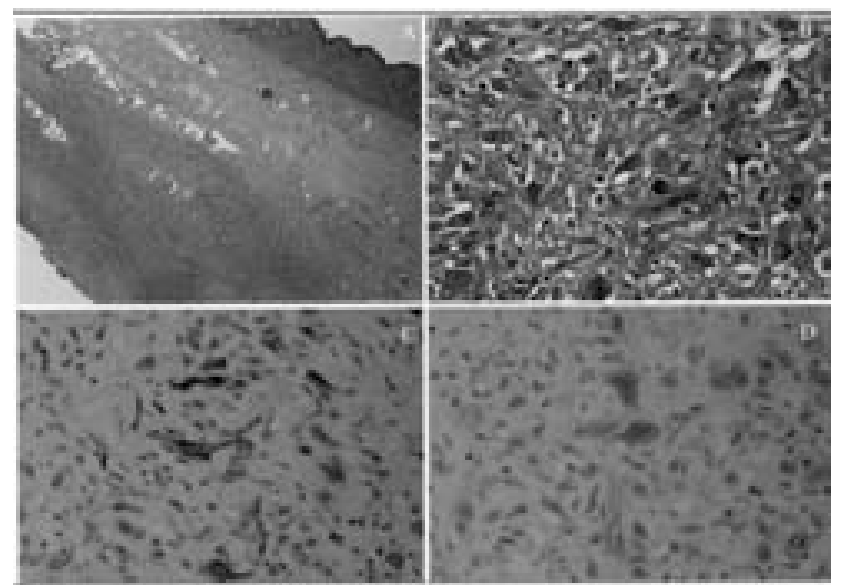

Figura 6. Anatomía patológica: (A) Imagen panorámica de la lesión con revestimiento externo formado por un epitelio pavimentoso queratinizante y revestimiento interno neoplásico (HE 4x). (B) Neoplasia de aspecto sarcomatoide formada por células fusiformes y poligonales con núcleos irregulares y frecuentes mitosis (HE 40x). (C) Células tumorales positivas para citoquinas (AEI - AE3 40x). (D) Células tumorales positivas para antígeno de membrana epitelial (EMA 40x).

comprobar que no existía salida de fluidos, no se continuó una disección intrarraquídea. Quedó un defecto dérmico importante que preciso reconstrucción por cirugía plástica, al no poder utilizar la propia piel del saco.

El análisis anatomopatológico de la pieza extirpada, demostró la existencia de un carcinoma de células escamosas sarcomatoide, secundario a la malignización de un quiste epidermoide malformativo de la disrafia. Un estudio inmuno-histoquímico reveló marcadores epiteliales positivos (AE1-AE3 y EMA) (Fig. 6).

Dos meses después presentó evolución rápida y progresiva de cefalea, pérdida de memoria, trastornos de conducta y diplopia. En los estudios generales no se encontró inicialmente ninguna causa justificable, ni tampoco en estudios repetidos de neuroimagen. Durante este proceso presentó un cuadro irritativo meníngeo afebril, que obligó a realizar mediante punción cisternal un análisis de LCR. Su estudio histopatológico indicó la existencia de una siembra celular carcinomatosa. El deterioro del paciente fue progresivo falleciendo a los 3 meses de este último diagnóstico.

\section{Discusión}

A partir de los años 70, en la literatura científica se empezaron a publicar series de casos que mostraban posibles asociaciones entre la espina bífida en sus distintas modalidades y diferentes tipos de tumores.

Es conocida la presencia del meningocele junto a 
tumores dermoides, epidermoides ${ }^{12,20}$, meningiomas, neurofibromas, cordomas, condromas, lipomas y en un alto porcentaje con teratomas. Incluso se conoce un caso publicado en que coinciden meningocele sacro con un teratocarcinoma intracraneal ${ }^{7}$.

Existe una agrupación sindrómica catalogada como Síndrome de Currarino, caracterizada por la existencia de múltiples anomalías caudales congénitas destacando malformaciones ano-rectales, anomalías del sacro y masas presacras $^{2,4,8}$.

Publicaciones posteriores de los años 80 sugieren una relación del tumor de Wilms (nefroblastoma) con pacientes afectos de espina bífida oculta o con estigmas cutáneos de disrafia espinal, pudiendo ser demostrada estadísticamente a comienzos de la década actual ${ }^{14}$.

La teoría más común que tiende a explicar estas conexiones tumorales con los casos de disrafia espinal, indica que la presencia de células multipotenciales del primordio neural, al ser activadas por los cambios endocrinológicos en la adolescencia, podrían facilitar la transformación maligna de estas lesiones, si no se han extirpado antes de la edad adulta ${ }^{15}$. Además, la existencia de una irritación crónica de la zona por una fístula de LCR puede favorecer dicha degeneración.

Los dos casos que aportamos tratan de pacientes que llegaron a la edad adulta sin haber sido intervenidos de sus sacos meningocélicos, desconociendo la existencia de tumores concomitantes con su proceso congénito y no habiendo tenido fístula licuoral.

En el caso del paciente $n^{\circ} 2$ se describe una malignización de un quiste epidermoide malformativo tras un proceso continuado de infección del saco meningocélico. En la literatura existen casos similares de transformación carcinomatosa de lesiones malformativas ya existentes, siendo característico el hecho de infecciones repetidas previas y de fístulas de líquido cefalorraquídeo. Concretamente hemos registrado 5 casos de malignización sobre el quiste malformativo o la lesión acompañante.

Thorp $^{21}$ (1967) presenta el caso un varón de 26 anos que desarrolló un carcinoma anaplásico sobre un mielomeningocele con drenaje crónico de LCR. a través de una fístula dérmica.

Love $^{10}$ (1968) aporta el caso de un varón de 30 años de edad con un meningocele lumbosacro que debutó con una masa inguinal derecha que correspondía con una metástasis linfática. La biopsia de la lesión lumbar alertó de la presencia de un teratoma maligno con extensas áreas de adenocarcinoma.

Mickle $^{15}$ (1975) publicó el caso de una niña de 11 años con meningocele asociado a un lipoma y que en los últimos meses antes de ser intervenida notó un endurecimiento de esa masa lumbo-sacra. Tras la inter- vención se demostró la existencia de un nefroblastoma con metástasis linfáticas.

Banerjee $^{1}$ (1977) describe el .caso de un mielomeningocele en una mujer de 20 años que tras su resección presenta continuas infecciones degenerando al final los bordes en un carcinoma de células escamosas con diseminación retroperitoneal.

Marini $^{11}$ (1979) relata el caso de un varón de 41 años que desarrolló un carcinoma espino celular sobre un pequeño meningocele con un tracto fistuloso que drenaba crónicamente LCR. Los estudios de imagen demostraron invasión de células carcinomatosas por el espacio subdural hasta la zona peduncular. A los pocos meses de la resección de la lesión, el paciente presentó una lesión nodular inguinal que fue diagnosticada como metástasis linfática.

Posteriormente se han encontrado tumores y quistes malformativos asociados a disrafias espinales, pero sin mostrar una transformación carcinomatosa del saco meningocélico.

Llama la atención el hecho de que todos los trabajos encontrados sean anteriores a los años 80. Pensamos que esto pudiera estar relacionado con los avances en el diagnostico prenatal y con la posibilidad posterior de interrumpir el embarazo, así como también por los avances quirúrgicos de las últimas tres décadas que nos han permitido tratar a los neonatos afectos de esta patología.

La evolución clínica del caso de Mickle tiene muchas similitudes con nuestro primer caso. Ambos pacientes convivieron perfectamente con sus lesiones hasta que empezaron a notar un aumento de volumen, de consistencia y de molestias de la lesión, motivos que obligaron a ser intervenidos.

En los casos de Thorp y de Marini, la irritación crónica por parte del LCR sobre la piel a través de su trayecto fistuloso pudiera ser la causante de la degeneración carcinomatosa de los tejidos. Estos casos semejan mucho a la evolución de nuestro segundo paciente, en el que aunque nunca existió ni se evidenció comunicación fistulosa de LCR, pudo existir en algún momento una entrada de células carcinomatosas a través del tracto fistuloso que mantendría comunicación con el espacio subaracnoideo espinal.

\section{Conclusión}

Con la aportación de nuestros dos casos intentamos recordar la tendencia que existe de malignización en los tejidos que recubren el saco meningocélico, aumentando el riesgo en los pacientes portadores de estas malformaciones con edades avanzadas.

Sugerimos como tratamiento de elección la exéresis quirúrgica de los meningoceles de forma precoz aunque no fistulicen y sean asintomáticos. En casos concretos como 
los cerrados y sin fístula, una estrecha vigilancia de la lesión sería también una opción valida.

La presencia de trayectos fistulosos de LCR obligaría a realizar cirugía y resecciones con amplios márgenes de seguridad en la resección del trayecto.

\section{Bibliografía}

1. Banerjee, T.: Nonhealing myelomeningecele in an adult. South Med J. 1977; 70: 367-368.

2. Currarino, G., Coln, D., Votteler, T.: Triad of anorectal, sacral and presacral anormalies. A.JR. 1981; 137: 395-398.

3. Dias, M.S., McLone, D.G.: Normal and abnormal early development of the Nervous System. En Pediatric Neurosurgery. Surgery of the developing nervous System, pp 31-71. Saunders Ed., 2001, New York.

4. Dios Seoane, J., Amaro, S., Fantini, M.G., et al.: Anterior sacral meningocele with Currarino's syndrome: report of two cases. Neurocirugía 2002; 13: 455-462.

5. Ersahin, Y., Barcin, E., Mutluer, S.: Is meningocele really an isolated lesion? Childs Nery Syst 2001; 17: 487490.

6. French, B.N.: Midline Fusion Defects and Defects of Formation. En Youmans J.R. Neurological Surgery; W.B. Saunders Company. 1982 ; pp1236-1372.

7. Holness, R.O., Hoffman, H.J., Mancer, K., et al.: Intracranial teratocarcinoma in a child with anterior sacral and intrasacral meningocele. Neurosurgery 1978; 2: 143-147.

8. Horii, T., Tsuchiya, H., Tomita, K.: Presacral tumor associated with the Currarino triad in an adolescent. Arch Orthop Trauma Surg. 2001; 121: 114-116.

9. Lober, J., Ward, A.M.: Spina Bifida — A Vanishig Nightmare? Arch Dis Chile. 1979; 60: 413-419.

10. Love, J.G.: Delayed malignant development of a congenital teratoma with spina bifida: Case report. J. Neurosurg. 1968; 29: 532-534.

11. Marini, G., Bollati, A., Galli, G., et al.: Carcinoma associated with lipomeningocele. Case report. Neurosurgery
1979; 5: 268-269.

12. Martinez-Lage, J.F., Masegosa, J., Sola, J., et al.: Epidermoid cyst occurring within a lumbosacral myelomeningocele. Case report. J Neurosurg 1983; 59: 1095-1097.

13. May, D., Rilliet, B., Berney, J.: Cervical meningocele and meningomyelocystocele. Apropos of 4 cases. Neurochirurgie 1992; 38: 347-352.

14. Mehes, K., Weisenbach, J., Kajtar, P.: Association of wilms tumor with spinal dysraphism. Pediatr Hematol Oncol. 2003; 20: 261-264.

15. Mickle, J.P., McLennan, J.E.: Malignant teratoma arising within a lipomeningocele. Case report. J Neurosurg 1975; 3: 761-763.

16. Oakley, G.P. Jr, Erickson, J.D., James, L.M., et al.: Prevention of folic acid-preventable spina bifida and anencephaly. Ciba Found Symp 1994; 212-223; discussion 223231.

17. Poza, M.: Cirugía del sistema nervioso y del raquis (fundamentos); Universidad de Murcia, 1993; pp. 252-259.

18. Sadler, T.W.: Langman. Embriología médica. Baltimore; Willianms \& Wilkins, 1991; pp. 345.

19. Sanchis Calvo, A., Martinez-Frias, M.: Clinical epidemiological study of neural tube defects classified according to the five sites of closure. An Esp Pediatr. 2001; 54: 165-173 .

20. Shamoto, H., Yoshida, Y., Shirane, R., et al.: Anterior sacral meningocele completely occupied by an epidermoid tumor. Childs Nery Syst 1999; 15: 209-211.

21. Thorp, R.H.: Carcinoma associated with myelomeningocele: Case report. J. Neurosurg. 1967; 27: 446-448.

Rivero-Celada, D.; Carcavilla-Loncán, L.I.; Marín-Cárdenas, M.A.; Cantero-Antón, J.M.; Alfaro-Torres J.; Duato-Jané, F.; Eiras-Ajuria, J.: Degeneración tumoral en meningocele no intervenido. Descripción de dos casos. Neurocirugía 2006; 17: 532-537.

Correspondencia postal: David Rivero Celada. C/ Eugenio Lucas 20, Esc. $1^{\mathrm{a}}, 3^{\circ}$ A. 50018 Zaragoza. 